\title{
First Concise Total Synthesis of Biologically Interesting Natural Licoagrochalcone $B$ and Its Unnatural Derivatives
}

\author{
Yong Rok Lee and Xue Wang \\ School of Chemical Engineering and Technology, Yeungnam University, Gyeongsan 712-749, Korea. "E-mail: yrlee@yuackr \\ Received September 17, 2007
}

Key Words : Natural product, Licoagrochalcone B, Pyranochalcone, $\mathrm{Nldol}$ reaction

Pyranochalcones are an abundant subclass of the flavonoid and are widely distributed in nature. ${ }^{1}$ Members of the pyranochalcones have been associated with a wide variety of biological activities such as antimutagenic, antimicrobial, anti-ulcer, and antitumor activities and some plants are used in traditional medicines in China and Europe. ${ }^{2}$ As part of an ongoing study into traditional medicine and pharmacological tests, the biologically interesting compound, licoagrochalcone $\mathrm{B}$ (1) with the pyranochalcone moiety was isolated from Patrinia villosa (BailiangCao in China) and Glvcurhiza glabrat (Figure 1). Licoagrochalcone B (1) shows potent anticancer activity against human cancer cell such as A549, BEL-7402, SGC-7901, MCF-7, HT-29, K562, and A $498{ }^{3 a}$ Patrinia species are distributed mainly in Central to East Asia and northeast North America. The ShenNongBenCooJing, a famous ancient Chinese medicinal literary, has documented their use as medicinal plants for more than 2000 years. Some of these plants are still used in traditional medicine as antiviral and antibacterial agents. ${ }^{3}$<smiles>[R]Oc1c(/C=C/C(=O)c2ccc([R3])c([R])c2[R1])ccc2c1C=CC(C)(C)O2</smiles>

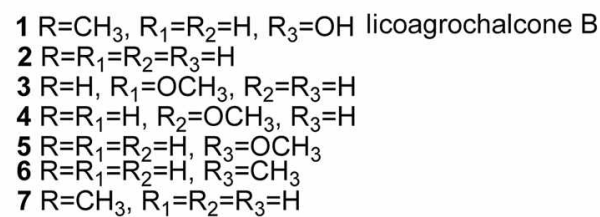

Figure 1. Naturally occurrng licoagrochalcone B (1) and unnatural its derivatives 2-7.
Licorice, the root and stolon of Glvcyrrhiza species, is also one of the oldest and important medicinal plants. ${ }^{4}$ It has been shown to have a variety of biological properties such as antimutagenic, anti-ulcer, antitumor, antioxitant, and antimicribial activities. "In addition, one of the fractions from the extract of licorice has been developed as an anti-ulcer drug (Aspalon) in Japan. ${ }^{4}$ This wide range of biological properties has stimulated interest in the synthesis of naturally occurring licoagrochalcone B (1) and its derivatives 2-7.

Recently, we reported the synthesis of natural products of lonchocarpin (12) and its derivative 13 with the pyranochalcone moiety starting from 1,3-cyclohexanedione (8) utilizing ethylenediamine diacetate-catalyzed $2 \mathrm{H}$-pyran formation as a key step (Scheme 1). ${ }^{6}$ However, although this lonchocarpin (12) and its derivative 13 contain pyranochalcone structure, they are different from licoagrochalcone B and its derivatives in the chalcone moiety. As an extension of our work to the synthesis of pyranochalcones, we report the first synthesis of a biologically interesting natural compound, licoagrochalcone $\mathrm{B}$ (1) along with its derivatives 2-7.

\section{Results and Discussion}

The retrosynthetic strategy of licoagrochalcone B (1) and its derivatives 2-7 is shown in Scheme 2. Licoagrochalcone $\mathrm{B}$ (1) and its derivatives $2-7$ could be prepared from a basecatalyzed aldol reactions of acetophenones 16-21 to benzopyran 15. The crucial intermediate 15 could be generated from the commercially available material 8 or 14 via ethylenediamine diacetate-catalyzed $2 H$-pyran or benzopyran formation reactions.

The benzopyran 15 was first achieved from 1,3-cyclohexanedione ( 8 ) in $50 \%$ overall yield ( 3 steps) using the

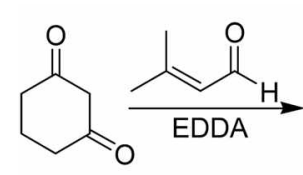

8<smiles>CC1(C)C=CC2=C(CCCC2=O)O1</smiles>

9

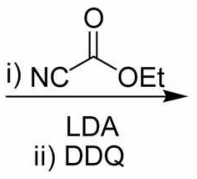

ii) $\mathrm{DDQ}$<smiles>CCOC(=O)c1ccc2c(c1O)C=CC(C)(C)O2</smiles>

10
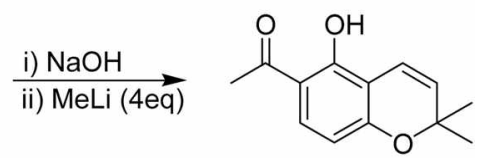

11

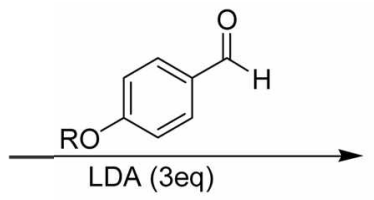<smiles>[R6]c1ccc(/C=C/C(=O)c2ccc3c(c2O)C=CC(C)(C)O3)cc1</smiles>

$12 \mathrm{R}=\mathrm{H}$ lonchocarpin $13 \mathrm{R}=\mathrm{OH}$ 4-hydroxylonchocarpin

Scheme 1 
<smiles>[R]Oc1c(/C=C/C(=O)c2ccc([R3])c([R])c2[R])ccc2c1C=CC(C)(C)O2</smiles><smiles>CC1(C)C=Cc2c(ccc(C=O)c2O)O1</smiles><smiles>CC1(C)C=CC2=C(CCCC2=O)O1</smiles><smiles>O=C1CCCC(=O)C1</smiles>

Scheme 2. Retrosynthetic analysis of licoagrochalcone (1) and its derivatives 2-7.

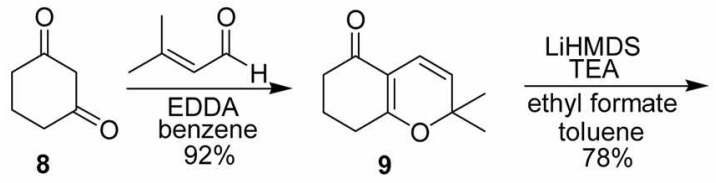

9

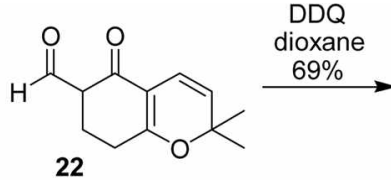

Scheme 3 methodology described previously, as shown in Scheme 3.? A reaction of compound 8 with 3-methyl-2-butenal in the presence of $10 \mathrm{~mol} \%$ of ethylenediamine diacetate in refluxing benzene for $3 \mathrm{~h}$ gave the adduct 9 in $92 \%$ yield. Treatment of compound 9 with ethyl formate in the presence of LiHMDS/TEA at $-78^{\circ} \mathrm{C}$ gave compound 22 in $78 \%$ yield. ${ }^{8}$ The oxidation of compound 22 with DDQ in refluxing dioxane afforded compound $\mathbf{1 5}$ in $69 \%$ yield. Recently, we also reported a new methodology for the preparation of a variety of benzopyrans by ethylenediamine diacetate-catalyzed reactions of substituted resorcinols or trihydroxybenzenes to $\alpha, \beta$-unsaturated aldehydes. ${ }^{9}$ These reactions involve a formal [3+3]-cycloaddition through a $6 \pi$-electrocyclization. To develop more convenient and efficient method for the preparation of benzopyran 15 , a one-step reaction was next attempted starting from 2,4-dihydroxybenzaldehyde (23). The reaction of compound 23 with 3-methyl2 -butenal in the presence of $10 \mathrm{~mol} \%$ of ethylenediamine diacetate in refluxing toluene for $10 \mathrm{~h}$ afforded the adduct 15 in $67 \%$ yield.

To complete the synthesis of licoagrochalcone B (1), an aldol reaction was next tried as shown in Scheme 4 . Attempts to condense 4-hydroxyacetophenone or protected compound 16 to benzopyran 15 using $\mathrm{KOH}$ in ethanol were unsuccessful. After examining many procedures, a reaction of compound 16 with benzopytan 15 using $\mathrm{NaH}$ in DMSO at room temperature for $48 \mathrm{~h}$ provided compound 24 in $71 \%$ yield. The assignment of $E$ stereochemistry of compound 24 was easily defined as $(E)$ by observation of vicinal coupling constants $(J=15.6 \mathrm{~Hz})$ of $\alpha, \beta$-unsaturated carbonyl group. Methylation of compound $\mathbf{2 4}$ with methyl iodide under
$\mathrm{K}_{2} \mathrm{CO}_{3}$ in acetone at room temperature for $10 \mathrm{~h}$ gave compound $25(96 \%) .{ }^{10}$ Deprotection of 2-(trimethylsilyl)ethoxymethyl (SEM) group of compound 25 using TBAF gave product 1 in low yield (38\%). Fortunately, reaction of 25 with $\mathrm{MgBr} 2 \cdot \mathrm{OEt}_{2}$ in dichloromethane at room temperature for $3 \mathrm{~h}$ afforded licoagrochalcone $\mathrm{B}(\mathbf{1})$ in high yield $(89 \%)$. The spectral data of compound $\mathbf{1}$ was in good agreement with that of the natural product reported in the literature. ${ }^{40}$

In order to extend the utility of this reaction, the synthesis of various analogues of compound 1 was also attempted through further aldol reactions as shown in Scheme 5. Reactions of compounds 17-21 with benzopyran 15 using $\mathrm{NaH}$ in DMSO at room temperature for $48 \mathrm{~h}$ gave products $2-6$ in $83,78,82,80$, and $83 \%$ yield, respectively. Reaction of compound 2 with methyl iodide under $\mathrm{K}_{2} \mathrm{CO}_{3}$ in acetone at room temperature for $10 \mathrm{~h}$ gave compound 7 in $94 \%$ yield. ${ }^{10}$

In conclusion, the synthesis of biologically interesting natural licoagrochalcone B (1) and its derivatives 2-7 was accomplished from commercially available 1,3-cyclohexanedione (8) or 2,4-dihydroxybenzaldehyde (23). The key strategy in the synthetic strategy involves the fornation of $2 \mathrm{H}$-pyran or benzopyran and an aldol reaction.

\section{Experimental Section}

2,2-Dimethyl-2,6,7,8-tetrahydrochromen-5-one (9). To a solution of 1,3 -cyclohexanedione $(8)(1.120 \mathrm{~g}, 10.0 \mathrm{mmol})$ and 3-methyl-2-butenal $(1.680 \mathrm{~g}, 20.0 \mathrm{mmol})$ in benzene ( 50 $\mathrm{mL}$ ) was added ethylenediamine diacetate $(180 \mathrm{mg}, \mathrm{mmol})$ at room temperature. The reaction mixture was refluxed for

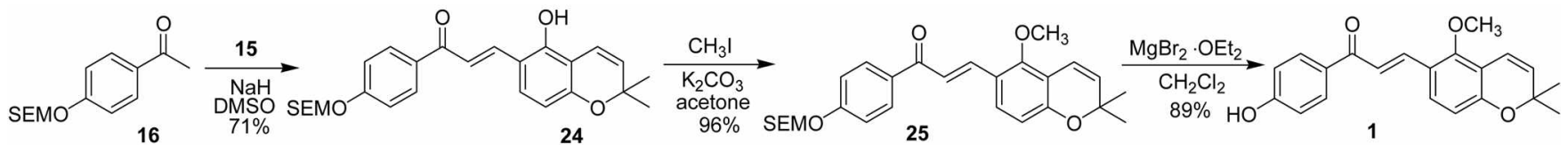

Scheme 4 

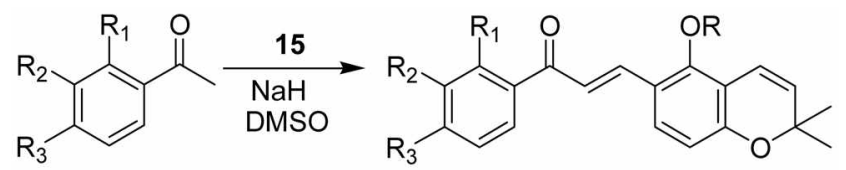

$17 \mathrm{R}_{1}=\mathrm{R}_{2}=\mathrm{R}_{3}=\mathrm{H}$ $18 \mathrm{R}_{1}=\mathrm{OCH}_{3}, \mathrm{R}_{2}=\mathrm{R}_{3}=\mathrm{H}$ $19 \mathrm{R}_{1}=\mathrm{H}, \mathrm{R}_{2}=\mathrm{OCH}_{3}, \mathrm{R}_{3}=\mathrm{H}$ $20 \mathrm{R}_{1}=\mathrm{R}_{2}=\mathrm{H}, \mathrm{R}_{3}=\mathrm{OCH}_{3}$ $21 \mathrm{R}_{1}=\mathrm{R}_{2}=\mathrm{H}, \mathrm{R}_{3}=\mathrm{CH}_{3}$

$83 \%$ $78 \%$ $82 \%$ $80 \%$ $83 \%$ $94 \%$

\section{Seheme 5}

$3 \mathrm{~h}$ and then cooled to room temperature. Water was added and the solution was extracted with ethyl acetate. Evaporation of solvent and purification by column chromatography on silica gel gave $9(1.639 \mathrm{~g}, 92 \%)$ as an oil: ${ }^{1} \mathrm{H}$ NMR $\left(300 \mathrm{MHz}, \mathrm{CDCl}_{3}\right) \delta 6.38(1 \mathrm{H}, \mathrm{d}, J=10.0 \mathrm{~Hz}), 5.2 \mathrm{l}$ $(1 \mathrm{H}, \mathrm{d}, J=10.0 \mathrm{~Hz}), 2.39-2.34(4 \mathrm{H}, \mathrm{m}), 1.99-1.90(2 \mathrm{H}, \mathrm{m})$, $1.37(6 \mathrm{H}, \mathrm{s}) ;{ }^{13} \mathrm{C}$ NMR $\left(75 \mathrm{MHz}, \mathrm{CDCl}_{3}\right) \delta 195.3,172.1$, $123.2,116.1,110.9,80.1,36.8,29.0,28.5,28.5,21.0$; IR (neat) $2926,1645,1611,1455,1399,1375,1266,1188$, $1130,1010,905 \mathrm{~cm}^{-1}$; HRMS m/z $\left(\mathrm{M}^{+}\right)$calcd for $\mathrm{C}_{11} \mathrm{H}_{14} \mathrm{O}_{2}$ : 178.0994. Found: 178.0996

2,2-Dimethyl-5-oxo-5,6,7,8-tetrahydro-2H-chromene6-carbaldehyde (22). To a stirred solution of $1,1,1,3,3,3$ hexamethyldisilazane ( $1.162 \mathrm{~g}, 7.2 \mathrm{mmol})$ in $30 \mathrm{~mL}$ of dry toluene was added a solution of $n$-BuLi $(2.5 \mathrm{M}, 2.6 \mathrm{~mL})$ in hexane at $-78^{\circ} \mathrm{C}$. After stirring at the same temperature for $30 \mathrm{~min}$, compound $9(0.570 \mathrm{~g}, 3.2 \mathrm{mmol})$ in toluene $(2 \mathrm{~mL})$ and TEA $(3.290 \mathrm{~g}, 32.5 \mathrm{mmol})$ were added through a cannula. After $30 \mathrm{~min}$, ethyl formate $(0.474 \mathrm{~g}, 6.4 \mathrm{mmol})$ in toluene $(1 \mathrm{~mL})$ was added. The reaction mixture was stirred at the same temperature for $3 \mathrm{~h}$, warmed to room temperature, quenched by addition of aqueous $\mathrm{NH}_{4} \mathrm{Cl}$ solution. The mixture was extracted with ethyl acetate $(3 \times 50 \mathrm{~mL})$. The combined organic layers were washed with brine, dried over $\mathrm{MgSO}_{4}$, and evaporated under reduced pressure. The residue was purified by flash column chromatography on silica gel to give 22 as an oil $(0.515 \mathrm{~g}, 78 \%)$ : $1 \mathrm{H}$ NMR $(300 \mathrm{MHz}$, $\left.\mathrm{CDCl}_{3}\right) \delta 13.78(1 \mathrm{H}, \mathrm{d}, J=11.0 \mathrm{~Hz}), 7.12(1 \mathrm{H}, \mathrm{d}, J=11.0$ $\mathrm{Hz}), 6.4(1 \mathrm{H}, \mathrm{d}, J=10.0 \mathrm{~Hz}), 5.27(1 \mathrm{H}, \mathrm{d}, J=10.0 \mathrm{~Hz}), 2.57-$ $2.35(4 \mathrm{H}, \mathrm{m}), 1.40(6 \mathrm{H}, \mathrm{s})$; IR (neat) $2978,1637,1586, \mathrm{I} 433$, $1358,1273,1225,1208,1134,1088,972,899 \mathrm{~cm}^{-1}$; HRMS $m / z\left(\mathrm{M}^{+}\right)$Calcd for $\mathrm{C}_{12} \mathrm{H}_{14} \mathrm{O}_{3}: 206.0943$. Found: 206.0941 .

5-Hydroxy-2,2-dimethyl-2H-chromene-6-carbaldehyde (15) from 22. A mixture of $22(0.455 \mathrm{~g}, 2.2 \mathrm{mmol})$ and DDQ $(0.749 \mathrm{~g}, 3.3 \mathrm{mmol})$ in dioxane $(30 \mathrm{~mL})$ was heated under reflux for $3 \mathrm{~h}$. The resulting mixture was cooled in an ice bath and solids were removed by filtration through Celite. The filtrate was evaporated under reduced pressure and purified by flash column chromatography on silica gel to give $15(0.310 \mathrm{~g}, 69 \%)$ as a solid: $m p 45-47^{\circ} \mathrm{C}$; H $\mathrm{HMR}$ $\left(300 \mathrm{MHz}, \mathrm{CDCl}_{3}\right) \delta \mathrm{l} 1.63(1 \mathrm{H}, \mathrm{s}), 9.64(1 \mathrm{H}, \mathrm{s}), 7.27(1 \mathrm{H}, \mathrm{d}$, $J=8.5 \mathrm{~Hz}), 6.67(1 \mathrm{H}, \mathrm{d}, J=10.1 \mathrm{~Hz}), 6.40(1 \mathrm{H}, \mathrm{d}, J=8.5$ $\mathrm{Hz}), 5.59(1 \mathrm{H}, \mathrm{d}, J=10.0 \mathrm{~Hz}), \mathrm{l} .54(3 \mathrm{H}, \mathrm{s}), \mathrm{l} .44(3 \mathrm{H}, \mathrm{s}) ;{ }^{13} \mathrm{C}$ NMR (75 $\left.\mathrm{MHz}_{,} \mathrm{CDCl}_{3}\right) \delta 194.9,161.0,159.1,135.1,129.0$, $115.6,115.5,109.8,109.2,78.6,29.8,28.8$; IR (KBr) 2974,
$1657,1580,1489,1433,1375,1335,1300,1256,1217$, $1181,1111,1088,974,937,900,845 \mathrm{~cm}^{-1}$; HRMS $m / z\left(\mathrm{M}^{+}\right)$ Calcd for $\mathrm{C}_{12} \mathrm{H}_{12} \mathrm{O}_{3}: 204.0786$. Found: 204.0788 .

5-Hydroxy-2,2-dimethyl- $2 \boldsymbol{H}$-chromene-6-carbaldehyde (15) from 23. To a solution of 2,4-dihydroxybenzaldehyde (23) (138 $\mathrm{mg}, 1.0 \mathrm{mmol}$ ) and 3-methyl-2-butenal (168 $\mathrm{mg}$, $2.0 \mathrm{mmol})$ in toluene $(20 \mathrm{~mL})$ was added ethylenediamine diacetate $(18 \mathrm{mg}, 1 \mathrm{mmol})$ at room temperature. The reaction mixture was refluxed for $10 \mathrm{~h}$ and then cooled to room temperature. Yield $67 \%(137 \mathrm{mg})$.

(E)-3-(5-Hydroxy-2,2-dimethyl-2H-chromen-6-yl)-1[4(2-trimethylsilanylethoxymethoxy)phenyl]propenone (24). To a solution of $16(160 \mathrm{mg}, 0.6 \mathrm{mmol})$ in DMSO $(10 \mathrm{~mL})$ was added sodium hydride $(72 \mathrm{mg}, 60 \%$ in oil, $1.8 \mathrm{mmol}$ ) and aldehyde 15 (143 $\mathrm{mg}, 0.7 \mathrm{mmol})$ at room temperature. The reaction mixture was stirred for $48 \mathrm{~h}$ at room temperature. Addition of water $(30 \mathrm{~mL})$ and extraction with ethyl acetate $(3 \times 50 \mathrm{~mL})$, washing with $2 \mathrm{~N}$-HCL solution and brine, drying over $\mathrm{MgSO}_{4}$ and removal of the solvent followed by flash column chromatography on silica gel gave $24(193 \mathrm{mg}, 71 \%)$ as a solid: $\mathrm{mp} 117-118^{\circ} \mathrm{C}$; $1 \mathrm{H}$ NMR $(300$ $\left.\mathrm{MHz}, \mathrm{CDCl}_{3}\right) \delta 8.52(\mathrm{lH}, \mathrm{d}, J=15.6 \mathrm{~Hz}), 8.02(2 \mathrm{H}, \mathrm{d}, J=$ $8.8 \mathrm{~Hz}), 7.47(\mathrm{lH}, \mathrm{d}, J=15.6 \mathrm{~Hz}), 7.46(1 \mathrm{H}, \mathrm{d}, J=8.6 \mathrm{~Hz})$, $7.09(2 \mathrm{H}, \mathrm{d}, J=8.8 \mathrm{~Hz}), 6.91(1 \mathrm{H}, \mathrm{d}, J=10.0 \mathrm{~Hz}), 6.42(1 \mathrm{H}$, $\mathrm{d}, J=8.6 \mathrm{~Hz}), 5.54(1 \mathrm{H}, \mathrm{d}, J=10.0 \mathrm{~Hz}), 5.28(2 \mathrm{H}, \mathrm{s}), 3.72$ $(2 \mathrm{H}, \mathrm{t}, J=8.6 \mathrm{~Hz}), 1.43(6 \mathrm{H}, \mathrm{s}), 0.95(2 \mathrm{H}, \mathrm{t}, J=8.6 \mathrm{~Hz})$, $-0.02(9 \mathrm{H}, \mathrm{s}) ;{ }^{13} \mathrm{C} \mathrm{NMR}\left(75 \mathrm{MHz}, \mathrm{CDCl}_{3}\right) \delta 190.4,161.7$, $157.2,153.5,141.6,132.4,131.2,129.4,128.2,118.2,117.0$, $116.4,116.2,110.6,110.2,93.0,77.7,76.9,30.1,28.4,18.5$ -0.99 ; IR (KBr) 2926, 1638, I60I, 1510, I464, 1233, 1173, $1092,992,837 \mathrm{~cm}^{-1}$; HRMS $m / z\left(\mathrm{M}^{+}\right)$Calcd for $\mathrm{C}_{26} \mathrm{H}_{32} \mathrm{O}_{5} \mathrm{Si}$ : 452.2019. Found: 452.2021 .

(E)-3-(5-Methoxy-2,2-dimethyl-2H-chromen-6-yl)-1-[4(2-trimethylsilanylethoxymethoxy)phenyl]propenone (25). Yield 96\%. H NMR $\left(300 \mathrm{MHz}, \mathrm{CDCl}_{3}\right) \delta 8.01(2 \mathrm{H}, \mathrm{d}, J=$ $8.8 \mathrm{~Hz}), 7.97(1 \mathrm{H}, \mathrm{d}, J=15.6 \mathrm{~Hz}), 7.50(\mathrm{lH}, \mathrm{d}, J=15.6 \mathrm{~Hz})$, $7.46(1 \mathrm{H}, \mathrm{d}, J=8.6 \mathrm{~Hz}), 7.09(2 \mathrm{H}, \mathrm{d}, J=8.8 \mathrm{~Hz}), 6.62(1 \mathrm{H}, \mathrm{d}$, $J=10.0 \mathrm{~Hz}), 6.59(\mathrm{lH}, \mathrm{d}, J=8.6 \mathrm{~Hz}), 5.65(\mathrm{IH}, \mathrm{d}, J=10.0$ $\mathrm{Hz}), 5.27(2 \mathrm{H}, \mathrm{s}), 3.77(3 \mathrm{H}, \mathrm{s}), 3.75(2 \mathrm{H}, \mathrm{t}, J=8.6 \mathrm{~Hz}), 1.43$ $(6 \mathrm{H}, \mathrm{s}), 0.94(2 \mathrm{H}, \mathrm{t}, J=8.6 \mathrm{~Hz}),-0.02(9 \mathrm{H}, \mathrm{s})$; IR (neat) 2955 , $1658,1603,1476,1424,1250,1169,1115,1094,988,837$ $\mathrm{cm}^{-1}$; EIMS m/z $466\left(\mathrm{M}^{+}, 10\right), 436(32), 435$ (100), 393 (49), 378 (25), $377(83), 218$ 924), 73 (64); HRMS $m / z\left(\mathrm{M}^{+}\right)$Calcd for $\mathrm{C}_{27} \mathrm{H}_{34} \mathrm{O}_{5} \mathrm{Si}: 466.2176$. Found: 466.2174 .

Licoagrochalcone B (1). To a solution of $25(100 \mathrm{mg}, 0.2$ $\mathrm{mmol})$ in $\mathrm{CH}_{2} \mathrm{Cl}_{2}(10 \mathrm{~mL})$ was added $\mathrm{MgBr}_{2} \cdot \mathrm{OEt}_{2}(207 \mathrm{mg}$, $0.8 \mathrm{mmol}$ ) and the mixture was stirred at room temperature for $3 \mathrm{~h}$. The reaction mixture was diluted with $\mathrm{CH}_{2} \mathrm{Cl}_{2}(50$ $\mathrm{mL}$ ), washed with saturated $\mathrm{NaHCO}_{3}$ solution $(30 \mathrm{~mL}$ ) and dried over $\mathrm{MgSO}_{4}$. After evaporation of the solvent under reduced pressure, the residue was purified by flash column chromatography on silica ge] to give $1(64 \mathrm{mg}, 89 \%)$ as a solid: mp 230-231 ${ }^{\circ} \mathrm{C}$; $\mathrm{H}$ NMR $\left(300 \mathrm{MHz}, \mathrm{CDCl}_{3}\right) \delta 7.98$ $(2 \mathrm{H}, \mathrm{d}, J=8.8 \mathrm{~Hz}), 7.94(1 \mathrm{H}, \mathrm{d}, J=15.6 \mathrm{~Hz}), 7.52(2 \mathrm{H}, \mathrm{d}, J$ $=15.6 \mathrm{~Hz}), 7.46(1 \mathrm{H}, \mathrm{d}, J=8.6 \mathrm{~Hz}), 6.94(2 \mathrm{H}, \mathrm{d}, J=8.8$ $\mathrm{Hz}), 6.62(\mathrm{lH}, \mathrm{d}, J=10.0 \mathrm{~Hz}), 6.60(1 \mathrm{H}, \mathrm{d}, J=8.6 \mathrm{~Hz}), 5.66$ $(1 \mathrm{H}, \mathrm{d}, J=10.0 \mathrm{~Hz}), 3.78(3 \mathrm{H}, \mathrm{s}), 1.44(6 \mathrm{H}, \mathrm{s}) ;{ }^{13} \mathrm{C} \mathrm{NMR}(75$ 
$\mathrm{MHz}$, acetone-d $\left.\mathrm{d}_{6}\right) \delta 188.2,162.6,157.1,138.2,131.8,131.7$, $131.6,129.2,121.9,121.3,117.2,116.2,116.1,115.8,113.8$, $77.4,63.2,28.2$; IR (KBr) 2924, 1601, 1462, 1373, 1262, $1074,802,741 \mathrm{~cm}^{-1}$; HRMS $m / z\left(\mathrm{M}^{+}\right)$Caled for $\mathrm{C}_{21} \mathrm{H}_{20} \mathrm{O}_{4}$ : 336.1362. Found: 336.1364 .

(E)-3-(5-Hydroxy-2,2-dimethyl-2H-chromen-6-yl)-1phenylpropenone (2). To a solution of 17 (48 $\mathrm{mg}, 0.4$ $\mathrm{mmol})$ in DMSO $(10 \mathrm{~mL})$ was added sodium hydride $(96$ $\mathrm{mg}, 60 \%$ in oil, $2.4 \mathrm{mmol}$ ) and aldehyde $15(102 \mathrm{mg}, 0.5$ mmol) at room temperature. The reaction mixture was stirred for $48 \mathrm{~h}$ at room temperature. Addition of water $(30$ $\mathrm{mL}$ ) and extraction with ethyl acetate $(3 \times 50 \mathrm{~mL})$, washing with $2 \mathrm{~N}-\mathrm{HCL}$ solution and brine, drying over $\mathrm{MgSO}_{4}$ and removal of the solvent followed by flash column chromatography on silica gel gave $2(102 \mathrm{mg}, 83 \%)$ as a solid: $\mathrm{mp}$ $112-113^{\circ} \mathrm{C}$; ${ }^{1} \mathrm{H}$ NMR $\left(300 \mathrm{MHz}, \mathrm{CDCl}_{3}\right) \delta 8.24(1 \mathrm{H}, \mathrm{d}, J=$ $15.6 \mathrm{~Hz}), 8.02-7.99(2 \mathrm{H}, \mathrm{m}), 7.59-7.42(5 \mathrm{H}, \mathrm{m}), 6.75(1 \mathrm{H}, \mathrm{d}$, $J=10.0 \mathrm{~Hz}), 6.45(1 \mathrm{H}, \mathrm{d}, J=8.6 \mathrm{~Hz}), 5.65(1 \mathrm{H}, \mathrm{d}, J=10.0$ $\mathrm{Hz}), 1.43(6 \mathrm{H}, \mathrm{s}) ;{ }^{13} \mathrm{C} \mathrm{NMR}\left(75 \mathrm{MHz}, \mathrm{CDCl}_{3}\right) \delta 191.9$, $157.3,153.3,141.9,138.9,133.2,129.7,128.9,128.7$, $118.8,116.7,116.1,110.5,110.4,77.9,28.4$; R (KBr) 3270, $2976,1644,1586,1564,1481,1352,1318,1209,1181$, $1123,1069,1042,1024,995,772 \mathrm{~cm}^{-1}$; EIMS m/z $306\left(\mathrm{M}^{+}\right.$, 25), $292(21), 291(100), 289(18), 275(23), 185(10), 105$ (88), $77(31), 69(12), 57(16), 55(14)$.

(E)-3-(5-Hydroxy-2,2-dimethyl-2H-chromen-6-yl)-1-(2methoxyphenyl)propenone (3). Yield $78 \%$. mp 126-127 ${ }^{\circ} \mathrm{C}$; 'H NMR (300 MHz, $\left.\mathrm{CDCl}_{3}\right) \delta 8.13(1 \mathrm{H}, \mathrm{d}, J=15.7 \mathrm{~Hz})$, 7.67-7.61 $(2 \mathrm{H}, \mathrm{m}), 7.44(1 \mathrm{H}, \mathrm{t}, J=8.6 \mathrm{~Hz}), 7.35(1 \mathrm{H}, \mathrm{d}, J=$ $15.7 \mathrm{~Hz}), 7.32(1 \mathrm{H}, \mathrm{d}, J=8.6 \mathrm{~Hz}), 7.03-6.95(2 \mathrm{H}, \mathrm{m}), 6.84$ $(1 \mathrm{H}, \mathrm{d}, J=10.0 \mathrm{~Hz}), 6.39(1 \mathrm{H}, \mathrm{d}, J=8.6 \mathrm{~Hz}), 5.59(1 \mathrm{H}, \mathrm{d}, J$ $=10.0 \mathrm{~Hz}), 3.97(3 \mathrm{H}, \mathrm{s}), 1.44(6 \mathrm{H}, \mathrm{s}) ;{ }^{13} \mathrm{C} \mathrm{NMR}(75 \mathrm{MHz}$, $\left.\mathrm{CDCl}_{3}\right) \delta 194.0,158.2,156.5,152.8,140.3,132.9,130.4$, $129.4,129.1,128.7,124.1,120.6,116.4,115.8,111.7,110.1$, $109.7,76.4,55.7,27.9$; IR (KBr) 2920, 1636, 1599, 1580, $1483,1464,1263,1117,1065,1034,810,739 \mathrm{~cm}^{-1}$; EIMS $\mathrm{m} / \mathrm{z} 336\left(\mathrm{M}^{+}, 27\right), 322(11), 321(52), 320$ (14), $319(14)$, $305(38), 207(12), 173(11), 135(100), 129(15), 77(12)$.

(E)-3-(5-Hydroxy-2,2-dimethyl-2H-chromen-6-yl)-1-(3methoxyphenyl)propenone (4). Yield 82\%. mp 135-136 ${ }^{\circ} \mathrm{C}$; 'H NMR (300 MHz, $\left.\mathrm{CDCl}_{3}\right) \delta 8.43(1 \mathrm{H}, \mathrm{d}, J=15.6 \mathrm{~Hz})$, $7.88(1 \mathrm{H}, \mathrm{s}), 7.60-7.53(2 \mathrm{H}, \mathrm{m}), 7.47-7.32(3 \mathrm{H}, \mathrm{m}), 7.09$ $(1 \mathrm{H}, \mathrm{d}, J=8.1 \mathrm{~Hz}), 6.86(1 \mathrm{H}, \mathrm{d}, J=10.0 \mathrm{~Hz}), 6.86(1 \mathrm{H}, \mathrm{d}, J$ $=8.6 \mathrm{~Hz}), 5.63(1 \mathrm{H}, \mathrm{d}, J=10.0 \mathrm{~Hz}), 3.83(3 \mathrm{H}, \mathrm{s}), 1.43(6 \mathrm{H}$, s) $;{ }^{13} \mathrm{C} \mathrm{NMR}\left(75 \mathrm{MHz}, \mathrm{CDCl}_{3}\right) \delta 191.9,160.2,157.5,153.6$, $142.4,140.3,129.9,129.5,128.5,121.6,119.8,118.5,116.9$, $116.2,113.3,110.6,110.3,77.4,55.8,28.4$; IR (KBr) 2975, $1635,1574,1464,1115,901,804,737 \mathrm{~cm}^{-1}$; EIMS m/z 336 $\left(\mathrm{M}^{+}, 30\right), 322(21), 321(100), 319(12), 305(20), 185(11)$, $135(69), 107(12)$.

(E)-3-(5-Hydroxy-2,2-dimethyl-2H-chromen-6-yl)-1-(4methoxyphenyl)propenone (5). Yield $80 \%$. mp 144-145 ${ }^{\circ} \mathrm{C}$; 'H NMR (300 MHz, $\left.\mathrm{CDCl}_{3}\right) \delta 8.48(1 \mathrm{H}, \mathrm{d}, J=15.6 \mathrm{~Hz})$, $8.02(2 \mathrm{H}, \mathrm{d}, J=8.8 \mathrm{~Hz}), 7.46(1 \mathrm{H}, \mathrm{d}, J=15.6 \mathrm{~Hz}), 7.45(1 \mathrm{H}$, d, $J=8.7 \mathrm{~Hz}), 6.97-6.92(3 \mathrm{H}, \mathrm{m}), 6.42(1 \mathrm{H}, \mathrm{d}, J=8.7 \mathrm{~Hz})$,
$5.62(1 \mathrm{H}, \mathrm{d}, J=10.0 \mathrm{~Hz}), 3.86(3 \mathrm{H}, \mathrm{s}), 1.43(6 \mathrm{H}, \mathrm{s}) ;{ }^{13} \mathrm{C}$ NMR (75 MHz, $\left.\mathrm{CDCl}_{3}+\mathrm{DMSO}_{-1}\right) \delta 190.2,163.8,157.1$, $153.4,141.2,131.7,131.3,129.4,128.4,118.5,117.0,116.4$, $114.2,110.6,110.2,76.9,55.9,28.3$; IR (KBr) 3175,2973 , $1634,1605,1582,1553,1350,1258,1235,1207,1165$, $1030,993,829,783,721 \mathrm{~cm}^{-1}$; EIMS m/z $336\left(\mathrm{M}^{+}, 22\right), 322$ (13), $321(60), 320(37), 319(33), 306(20), 305(95), 173$ (27), $135(100), 129(17), 83(16), 73(18), 71(19), 69(21)$, $57(29), 55(22)$.

(E)-3-(5-Hydroxy-2,2-dimethyl-2H-chromen-6-yl)-1-(4methylphenyl)propenone (6). Yield $83 \% . \mathrm{mp} 141-142^{\circ} \mathrm{C}$; 'H NMR $\left(300 \mathrm{MHz}, \mathrm{CDCl}_{3}\right) \delta 8.30(1 \mathrm{H}, \mathrm{d}, J=15.6 \mathrm{~Hz})$, $7.92(2 \mathrm{H}, \mathrm{d}, J=8.8 \mathrm{~Hz}), 7.46(1 \mathrm{H}, \mathrm{d}, J=15.6 \mathrm{~Hz}), 7.42(1 \mathrm{H}$, d, $J=8.6 \mathrm{~Hz}), 7.26(2 \mathrm{H}, \mathrm{d}, J=8.8 \mathrm{~Hz}), 6.82(1 \mathrm{H}, \mathrm{d}, J=10.0$ $\mathrm{Hz}), 6.43(1 \mathrm{H}, \mathrm{d}, J=8.6 \mathrm{~Hz}), 5.64(1 \mathrm{H}, \mathrm{d}, J=10.0 \mathrm{~Hz}), 2.41$ $(3 \mathrm{H}, \mathrm{s}), 1.43(6 \mathrm{H}, \mathrm{s}) ;{ }^{13} \mathrm{C}$ NMR $\left(75 \mathrm{MHz}, \mathrm{CDCl}_{3}+\mathrm{DMSO}-\right.$ $\left.\mathrm{d}_{6}\right) \delta 190.7,156.9,153.7,143.5,141.1,136.6,129.6,129.2$, $129.1,128.9,119.4,117.4,116.8,111.0,110.0,76.7,28.3$, 22.1; IR (KBr) 2973, 1634, 1610, 1283, 1115, 819, $737 \mathrm{~cm}^{-1}$; EIMS m/z $320\left(\mathrm{M}^{+}, 25\right), 306(17), 305(81), 303(11), 289$ (15), $185(11), 129(40), 119(100), 112(10), 91(26), 73$ (12), $71(14), 70(12), 69(21), 57(20), 55$ (14).

(E)-3-(5-Methoxy-2,2-dimethyl-2H-chromen-6-yl)-1phenylpropenone (7). Yield $94 \%$. ${ }^{1} \mathrm{H}$ NMR $(300 \mathrm{MHz}$, $\left.\mathrm{CDCl}_{3}\right) \delta 8.02-7.97(3 \mathrm{H}, \mathrm{m}), 7.55-7.45(5 \mathrm{H}, \mathrm{m}), 6.62(1 \mathrm{H}, \mathrm{d}$, $J=8.6 \mathrm{~Hz}), 6.59(1 \mathrm{H}, \mathrm{d}, J=10.0 \mathrm{~Hz}), 5.66(1 \mathrm{H}, \mathrm{d}, J=10.0$ $\mathrm{Hz}), 3.78(3 \mathrm{H}, \mathrm{s}), 1.44(6 \mathrm{H}, \mathrm{s})$; IR (neat) $2976,1658,1589$, $1476,1372,1296,1252,1208,1115,1073,988,889,818$, $775 \mathrm{~cm}^{-1}$; EIMS m/z $320\left(\mathrm{M}^{+}, 10\right), 306(21), 305(100), 290$ (21), 289 (99), 274 (10), 185 (44), $105(15), 77$ (15).

Acknowledgements. This work was supported by grant No. RTI04-01-04 from the Regional Technology Innovation Program of the Ministry of Commerce, Industry, and Energy (MOCIE).

\section{References and Notes}

I. Wagner, H.; Farkas, L. In The Flanonoids; Harome, J. B., Mabry, T. J., Mabry, H., Eds.; Academic Press: New York, 1975; p 127.

2. Welton, A. F.; Tobias, L. D.; Fiedler-Nagy, C.; Anderson, W; Hope, W.; Meyers, K.; Coffey, J. W. In Plant Flannoids in Biology and Medicine; Cody, V.; Middleton, Jr., E.; Harborne, J. B., Eds.; Alan R. Liss: New York, 1986; p 23 I.

3. (a) Peng, J.; Fan, G.; Wu, Y. J. Chromatogr. A 2006, 1115, 103. (b) Peng, J.; Fan, G.; Chai, Y.; Wu, Y.J. Chromatogr: A 2006, 1102, 44. 4. (a) Li, W.; Asada, Y.; Yoshikawa, T. Phytochemistry 2000, 55, 447. (b) Asada. Y.; Yoshikawa. T. Phtochenistry 1998, 47, 389.

5. Ngo, H. N.; Teel. R. W.; Lau, B. H. S. Nutrition Res. 1992, I2, 247.

6. Lee, Y. R.; Kim, D. H. Synthesis 2006, 603.

7. Lee, Y. R, Lee, W. K.; Noh, S. K.; Lyoo, W. S. Symthesis 2006, 853.

8. MeNeil, A. J.; Collum, D. B. J. Am, Chem. Soc, 2005, 127, 5655.

9. (a) Lee, Y. R.; Choi, J. H.; Yoon, S. H. Tetrahedron Letf. 2005, 46 , 7539. (b) Lee, Y. R.; Kim, J. H. Synlett 2007, 2232. (c) Lee, Y. R.; Wang X. Bull. Korean Chem. Soc. 2005, 26, 1933.

10. Shrestha, S.; Hwang, S. Y.; Lee, K.-H.; Cho, H. Bull. Korean Chem. Soc. 2005, 26, 1138. 